\title{
Medición de resultados en evaluaciones económicas
}

\author{
SANDRA SALDIVIA ${ }^{1, \mathrm{a}}$, BENJAMÍN VICENTE ${ }^{1}$, FRANCISCO TORRES $^{2}$
}

\section{Measuring outcomes in economic evaluations}

Beginning with the concept of evaluation, several approaches to outcomes or results evaluation are reviewed: clinical measurement, quality of life, and willingness to pay. Clinical measurement is the simplest and most available, but is limited when 1) establishing the effects of comparable interventions for different pathologies, 2) detecting different degrees of improvement achieved, and 3) identifying the result of interventions measured in variables associated to patient lifestyle and quality of life. When the health services measure the quality of life, the results of the intervention are subjectively evaluated by the patient with respect to wellbeing and satisfaction. The measurement of quality of life can be considered specific, generic or based on utility to the extent that it is based on the estimation of a specific variable such as the perception of pain, the patient's general state of health, or the estimation of uses normative values based in preference studies at the population level, respectively. The method of willingness to pay values the life according to the amount that individuals are willing to pay for a change that reduces the possibility of sickness and death, assuming that patients prefer those procedures to which they assigned the highest value. This evaluation of outcomes incorporates more than just strictly health considerations and expresses results in monetary terms, which facilitates cost-benefit analysis. Additionally, the results can be measured at a macro, national level; at a meso-scale, referring to the health systems of a determined territory; and at the level of the users. Finally, evaluating results or outcomes in order to determine the intervention's effectiveness should be associated to its cost and must be studied using research designs that guarantee that the changes have sufficient time to appear and are observables. The monitoring period should be sufficiently long to explain the different results observed in the studies.

(Rev Med Chile 2010; 138 (Supl 2): 79-82).

Key words: outcome research, economic evaluation, health-related quality of life.

\section{RESUMEN}

A partir del concepto de evaluación, se revisan distintas aproximaciones a los procesos de evaluación de resultados. Éstas incluyen la medición desde el punto de vista clínico, de calidad de vida, y de disponibilidad de pago. El punto de vista clínico resulta más simple y disponible, pero limitado al momento de establecer efectos de intervenciones comparables entre distintas patologías, de detectar los distintos grados de "mejoría" alcanzados y de dar cuenta del resultado de intervenciones medidas en variables asociadas al estilo y calidad de vida de los pacientes. Desde la perspectiva de calidad de vida relativa a la salud se busca evaluar los resultados que las intervenciones sanitarias tienen sobre la percepción subjetiva relacionada con bienestar y satisfacción. La calidad de vida puede ser considerada especifica, genérica o basada en la utilidad. En el primer caso, la medida se basa en una variable específica, como la percepción de
${ }^{1}$ Departamento de Psiquiatría y Salud Mental, Facultad de Medicina, Universidad de Concepción. ${ }^{2}$ Sección Departamental de Psiquiatría y Psicología Médica, Facultad de Medicina, Universidad de Granada. España.

apsicóloga, Doctora en Psicología.

Correspondencia a: Sandra Saldivia

Departamento de Psiquiatría y Salud Mental, Facultad de Medicina, Universidad de Concepción; Casilla 160-C, Concepción, Chile. Fax: (41) 2312799 E-mail: ssaldivi@udec.cl 
dolor; si la aproximación es genérica apunta al estado de salud general del paciente; y si se basa en la utilidad usa valores normativos basados en estudios de preferencia de nivel poblacional. La aproximación de disponibilidad de pago permite valorar la vida según lo que los individuos están dispuestos a pagar por un cambio que reduce la probabilidad de enfermedad o la muerte, asumiendo que aquellos procedimientos a los que se asigna mayor valor son los que el usuario prefiere. Permite incorporar valores que van más allá de lo sanitario y expresar resultados en términos monetarios, lo que facilita el análisis de costo-beneficio. Desde otra perspectiva, las medidas de resultados pueden incluir un nivel macro, nacional; una perspectiva meso, referida a los sistemas sanitarios de un determinado territorio; y la perspectiva de los usuarios. Finalmente, el estudio de resultados como derivado de la efectividad de la intervención debe asociarse al costo de ésta y ha de ser estudiado usando diseños de investigación que aseguren un tiempo de seguimiento suficiente que garantice que los cambios puedan ocurrir y sean observables. La longitud del seguimiento explica las diferencias observadas en los resultados de algunos estudios.

Palabras clave: Resultados en salud, evaluación económica, calidad de vida relativa a la salud.

$\mathrm{D}$ e acuerdo a Murray y Frenk ${ }^{1}$ el concepto de evaluación refiere a la disciplina que busca establecer una conexión causal entre una intervención y su efecto, lo que permite determinar la efectividad, eficiencia y aceptabilidad de la intervención planificada para alcanzar unos objetivos establecidos ${ }^{2}$. Esta evaluación puede orientarse al proceso, a través del seguimiento continuo; o al resultado, mediante la obtención de medidas de resultado (outcome).

Es esta última, la evaluación de resultados, la que permite comparar intervenciones cuando las unidades "producidas" son similares. Desde esta perspectiva, Torgenson y Raftery ${ }^{3}$ identifican tres aproximaciones principales: aquellos resultados medidos desde el punto de vista clínico, la medición de calidad de vida, y la disponibilidad de pago.

La medición desde el punto de vista clínico resulta en una unidad más simple y disponible, al menos teóricamente. Ejemplo de ella puede ser el número de pacientes que evoluciona positivamente frente a un cuadro obstructivo a partir de una intervención bien establecida a través de protocolos. En estos casos es posible medir el costo de la intervención y la efectividad de ella, pero también podrían evaluarse los costos evitados al comparar ésta respecto de otra intervención.

No obstante, en la práctica, medir resultados desde el punto de vista clínico resulta limitado dado que no es fácil establecer efectos de una intervención clínica que sean comparables entre distintas patologías, aún cuando en ello se incluya la mortalidad. La cada vez más utilizada medida de "años de vida ajustados por discapacidad" (DALY), que se revisa con mayor detención en otro artículo de esta serie, puede resultar útil para obtener información de costo-efectividad desde el punto de vista poblacional, pero tiene la debilidad del juicio de experto y, cuando se trata de ensayos clínicos, evidencia las limitaciones para detectar los distintos grados de "mejoría" alcanzados, y nada dicen acerca de la calidad de vida que acompañará a esos pacientes. Además, cuando se trata de patologías crónicas, las medidas de resultado clínico resultan limitadas para dar cuenta de intervenciones que se orientan a aspectos asociados a los estilos y calidad de vida de los pacientes. En estos últimos casos medidas psicosociales, como la de calidad vida, pueden responder mejor a los niveles de evolución que experimentan los pacientes tras una determinada intervención.

El concepto de calidad de vida tiene un doble origen. Uno procede de la sociología donde se ha usado como indicador complementario a los indicadores económicos que reflejaran el desarrollo positivo de las condiciones de vida de las poblaciones. El otro nace de la evidencia del ejercicio sanitario de que el aumento en la longitud de la vida no era siempre el objetivo único, ni siquiera el prioritario a veces, a la hora de evaluar los resultados de las intervenciones sanitarias, desde esta perspectiva se opta por referir a la "calidad de vida relativa a la salud", buscando operacionalizar una variable de resultado.

Lo anterior pone en evidencia que al hablar de calidad de vida se está indagando sobre una 
percepción subjetiva íntimamente relacionada con las nociones de bienestar y satisfacción y, en consecuencia, con una definición positiva de salud ${ }^{4}$.

Desde esta perspectiva, la calidad de vida puede ser una condición específica, genérica o basada en la utilidad ${ }^{3}$.

En el primer caso, el concepto remite a la experiencia subjetiva acerca de una variable específica, como el dolor, y, en consecuencia, los instrumentos de medición comprenderán preguntas particularmente referidas a aquello que la intervención busca resolver, como el estudio de la disminución del dolor usando la Escala del Dolor de Roland y Morris.

Cuando se la trata como una medida genérica, las preguntas apuntarán al estado de salud general del paciente, como la medición que se realiza usando el SF-36. Esta escala, de 36 ítems, evalúa 8 conceptos de salud, los que fueron elegidos por su amplio uso en los estudios de salud o por su fuerte soporte empírico, e incluyen: funcionamiento físico, limitaciones en el rol por problemas físicos de salud, funcionamiento social, dolor corporal, salud mental, limitaciones en el rol por problemas personales o emocionales, vitalidad y salud general. Las 8 dimensiones se agrupan en dos factores y subescalas, salud física y salud mental. El instrumento proporciona, además, un perfil del estado de salud, basado en la puntuación alcanzada en cada una de las 8 escalas 5 .

La medida de utilidad va más allá y buscará valores normativos basados en estudios de preferencia de nivel poblacional, desde esa perspectiva es una medida de preferencias societales y el EuroQoL EQ-5D, que permite puntuar preferencias respecto de 14 estados de salud posibles, es un instrumento frecuentemente utilizado ${ }^{6}$. Ello facilita la medición y comparación de resultados entre diferentes intervenciones, pero, en contraposición, puede ser poco sensible a la detección de cambios en el estado de salud, a menos que las muestras sean muy grandes, por lo cual se recomienda su uso en conjunto con otro tipo de medidas de resultado.

En ambos casos la percepción hace referencia a la experiencia de enfermar, al malestar, a aquello que en inglés se denomina illness en contraposición al concepto de disease que alude a las características clínicas del cuadro. A esta experiencia contribuyen variables como la percepción de los síntomas, la forma de entender y comunicar la vivencia de enfermedad, la vivencia de discapacidad o disminución de su nivel de funcionamiento habitual, con sus repercusiones a nivel familiar, social e incluyendo aspectos específicos como el ámbito laboral, y las estrategias desplegadas para conseguir algún tipo de control sobre el trastorno ${ }^{4}$.

En la misma línea de medición de preferencias en salud, el "conjoint analisys", aparece como uno de los métodos rigurosos para recolectar preferencias sociales y cuyo uso ha sido transferido desde los estudios de marketing al ámbito sanitario. Este método permitiría estimar la importancia relativa de los diferentes aspectos del cuidado y el grado de satisfacción global, o utilidad, que las personas derivan de los servicios sanitarios?

La técnica se basa en la premisa de que cualquier bien o servicio puede ser descrito por sus características o atributos y que la valoración que una persona hace de ese bien o servicio dependerá de estas características. Éstas deben ser identificadas; valoradas -asignando niveles cardinales, ordinales o categoriales-; organizadas dando lugar a diferentes escenarios entre los cuales los consultados puedan elegir y establecer sus preferencias, las que son indagadas en cuestionarios; para realizar finalmente un análisis de datos usando técnica de regresión.

La aproximación de disponibilidad de pago es otro método que permite valorar la vida según lo que los individuos están dispuestos a pagar por un cambio que reduce la probabilidad de enfermedad o la muerte; asumiendo que aquellos procedimientos a los que se asigna mayor valor son los que el usuario prefiere. Además de incorporar valores que van más allá de lo sanitario ésta técnica permite expresar esos resultados en términos monetarios, con lo cual facilita el análisis de costo-beneficio ${ }^{3}$.

Desde otra perspectiva, Murray y Frenk ${ }^{1}$ mencionan en un reciente artículo que las medidas de resultados en salud constituyen un área clave para la evaluación. Puntualizan además que lo que ellos denominan metrics en salud, que reúne los aspectos de medición, instrumentos e indicadores, proveen bases empíricas para entender un objeto particular de investigación y acción como lo es, en este caso, la salud global.

Desde esa perspectiva, señalan medidas de resultados de nivel macro, nacional, como son los indicadores de mortalidad, causas de muerte, incidencia, prevalencia y carga de las enfermedades y evaluación comparativa de riesgo. Pero desde una 
perspectiva meso, referida a los sistemas sanitarios responsables de la salud de la población de un determinado territorio, el equivalente nacional a los Servicios de Salud, las medidas de resultado han de ajustarse a las necesidades de los gestores locales y podrían incluir estudios de costo-efectividad y resultados de grupos de pacientes sujetos a determinadas características.

Desde la perspectiva de los usuarios, las medidas de resultados apuntarán a variables que den cuenta de su propia evolución incorporando medidas de calidad de vida, necesidades satisfechas, discapacidad, síntomas y medidas clínicas en general, satisfacción usuaria y, en aquellas patologías crónicas y/o con un componente psicosocial evidente, podrían incorporar la evaluación del efecto que ella tiene sobre la red primaria del paciente. Las medidas de carga del cuidador buscan responder a esa demanda.

De acuerdo a Häfner y An der Heiden ${ }^{8}$, los procesos de evaluación en salud mental, lo que puede ser transferido a la evaluación de resultados en salud en general, se ven dificultados por la presencia de fenómenos como las múltiples situaciones asistenciales y la complejidad de las intervenciones, las dificultades para encontrar criterios e indicadores de resultados apropiados, las dificultades de la medición y el control de las variables relevantes, y la existencia de prácticas diversas que generan diferentes métodos y prácticas para la recolección de los datos.

Frente a tales dificultades, el seguimiento y evaluación continua de servicios viene a constituirse en un procedimiento útil en las nuevas redes asistenciales. Del mismo modo, diseños de investigación basados en una aproximación desde abajo hacia arriba (bottom-up) proveen de información más diversificada y empírica en relación a la utilización de servicios y características psicopatológicas y sociales de los usuarios. Pero ambos modelos comparten algunas limitaciones.

Un resultado frecuentemente identificado en las referencias de los trabajadores de salud en relación a la evaluación de resultados es la medición de cambios en el nivel de productividad de los pacientes, lo que puede ser operacionalizado a través de variables como su ingreso y/o re-ingreso al mercado laboral. Pero ésta es un área controvertida dado que la evolución de los pacientes hacia el logro de este resultado no responde a una relación lineal sino que está sujeta a variables relacionadas con las características del cuadro y aún con las características del mercado laboral. Se estima que en el caso de pacientes más leves, pero que padecen una patología crónica, un manejo clínico eficiente debería aumentar su probabilidad de reingreso al mundo laboral, dado el mejoramiento en el funcionamiento clínico y social; pero no ocurriría lo mismo en el caso de pacientes más graves ${ }^{8}$. No obstante, tales hipótesis requieren ser evaluadas.

Por otra parte, el estudio de resultados como derivado de la efectividad de la intervención debe asociarse al costo de ésta y en tal sentido ha de ser estudiado usando diseños de investigación que aseguren un tiempo de seguimiento suficiente que garantice que los cambios puedan ocurrir y sean observables. La longitud del seguimiento explica las diferencias observadas en los resultados de algunos estudios de fármaco-economía y dan cuenta de la conveniencia de estudios de largo plazo donde deben considerarse además las probables consecuencias acarreadas de las pérdidas de pacientes y la manera de tratar tales problemas?

\section{Referencias}

1. Murray C, Frenk J. Health metrics and evaluation: strengthening the science. Lancet 2008; 371: 1191-9.

2. Holland WW. (Ed.) Measurement in health care. $1^{\text {a }}$ ed. Oxford: Oxford University Press; 1983.

3. Torgenson D., Raftery J. Measuring outcomes in economic evaluations. BMJ 1999; 318: 1413.

4. Baca E. Calidad de vida y aspectos económicos de la psiquiatría. El análisis coste-utilidad. Archivos de Neurobiología 1995; 58 (2): 189-217.

5. Ware J, Sherbourne CD. The MOS 36-Items Short-Form Health Survey. Medical Care 1992; 30 (6): 473-83.

6. Brooks R. EuroQoL: The current state of play. Health Policy 1996; 37 (1): 53-72.

7. Ryan M, Farrar S. Using conjoint analysis to elicit preferences for health care. BMJ 2000; 320: 1530-3.

8. Häfner H, An der Heiden W. Background and goals of evaluative research in community psychiatry. En Knudsen H. y Thornicroft G. (Eds.) Mental Health Services Evaluation. 1 $1^{\text {a }}$ ed. Cambridge: Cambridge University Press; 1996. p. 19-36.

9. Drummond MF, Knapp M, Burns TP, Miller KD, Shadwell P. Issues in the design of studies for the economic evaluation of new atypical antypsychotics: The ESTO study. The Journal of Mental Health Policy and Economics 1998; 1: 15-22. 